The Astrophysical Journal, 227:499-509, 1979 January 15

(C) 1979. The American Astronomical Society. All rights reserved. Printed in U.S.A.

\title{
INFRARED COLORS, CO BAND STRENGTHS, AND PHYSICAL PARAMETERS FOR GIANTS IN M71
}

\author{
JAY A. FrogeL* \\ Cerro Tololo Inter-American Observatory $\dagger$ \\ S. E. Persson \\ Hale Observatories, Carnegie Institution of Washington, California Institute of Technology \\ AND \\ JUDITH G. COHEN \\ Kitt Peak National Observatory $\dagger$ \\ Received 1978 May 1; accepted 1978 July 26
}

\begin{abstract}
Broad-band $J, H$, and $K$ magnitudes and narrow-band $\mathrm{CO}$ and $\mathrm{H}_{2} \mathrm{O}$ indices for 25 giant and horizontal-branch stars in the strong-lined globular cluster M71 are presented. These data are compared with similar data for M67 and for metal-poor globular clusters. In most photometric indices the M71 stars are intermediate between M67 and the metal-poor globulars, although the M71 giant branch extends to considerably cooler temperatures. Bolometric magnitudes and effective temperatures determined for the M71 stars also place this cluster between the other globulars and M67. A theoretical analysis of the CO indices indicates that M71 is metal deficient by $0.4( \pm 0.2)$ dex in the mean with respect to field giants.

The relationship between the $\mathrm{CO}$ indices and $\mathrm{CN}$ absorption strengths as determined from DDO photometry is as expected from empirically determined temperature dependences and theoretically determined abundance dependences.
\end{abstract}

Subject headings: clusters: globular — stars: abundances - stars: Population II

\section{INTRODUCTION}

This paper continues our program of infrared photometric observations of cluster stars (Cohen, Frogel, and Persson 1978, hereafter CFP). In CFP we presented data for giants in the metal-poor globulars M3, M13, and M92 and in the old disk open cluster M67. Here we present broad-band $J H K$ data and intermediate-band $\mathrm{CO}$ and $\mathrm{H}_{2} \mathrm{O}$ strengths for the brighter giants and some horizontal-branch stars in M71 (NGC 6838). From northern hemisphere sites, M71 is the most easily observable, strong-lined globular cluster (Morgan 1959). An extensive discussion of the $U B V$ color-color and color-magnitude diagrams has been given by Arp and Hartwick (1971, hereafter AH). More recently, Hesser, Hartwick, and McClure (1977, hereafter HHM) presented observations on the DDO system of several of the giants in M71, and Mould and McElroy (1978) reported on observations of the $7120 \AA \mathrm{TiO}$ band. Searle and Zinn (1978) gave measurements of a number of reddeningindependent metallicity indices in the blue.

Sections II, III, and IV describe the observations and present the physical parameters derived from them. In $\S \mathrm{V}$ the M71 data are compared with those of the other clusters. Section VI describes a quantitative

* Guest Investigator at the Hale Observatories.

$\uparrow$ Operated by the Association of Universities for Research in Astronomy, Inc., under contract with the National Science Foundation. technique for deriving $\mathrm{C}$ and $\mathrm{O}$ abundances from the $\mathrm{CO}$ indices and presents a comparison of the infrared data with DDO photometric data; $\S$ VII summarizes the conclusions.

\section{OBSERVATIONS}

The observations were made with the $5 \mathrm{~m}$ Hale reflector in 1977 September. The InSb detecting system and standard stars which define the photometric system have been described elsewhere (CFP; Frogel et al. 1978, hereafter Paper I; Aaronson, Frogel, and Persson 1978, hereafter Paper II). Seeing images which were typically $1^{\prime \prime}$ permitted the use of focal-plane apertures with projected diameters of $10^{\prime \prime} .0$ or $7 " .5$ for all of the observations. Because of the crowded nature of the field, we could not always eliminate background stars, which were 4 or 5 mag fainter than the program stars, from both signal and reference beams. Nevertheless, we believe the major source of error to be short-time-scale fluctuations in atmospheric transparency which resulted in poorer-than-normal repeatability of our standards. This is reflected in the errors noted in Table 1.

Table 1 contains the observed $J-H$ and $H-K$ colors and $K$ magnitudes of the M71 stars observed. All stars on the giant branch (GB), asymptotic giant branch (AGB), and horizontal branch (HB) observed photoelectrically by AH (their Table 1) which could be observed by us without confusion problems were 
TABLE 1

M71 РнOTOMETRY

\begin{tabular}{|c|c|c|c|c|c|c|c|c|c|c|c|c|}
\hline \multirow[b]{2}{*}{ star } & \multicolumn{3}{|c|}{ Observed* } & \multicolumn{8}{|c|}{ Reddening Correctedt } & \multirow[b]{2}{*}{ Notes } \\
\hline & $\bar{K}$ & $J-H$ & $\overline{H-K}$ & $K_{0}$ & $(U-V)_{0}$ & $(B-V)_{0}$ & $(V-K)_{0}$ & $(J-H)_{0}$ & $(H-K)_{0}$ & $\mathrm{CO}$ & $\mathrm{H}_{3} \mathrm{O}$ & \\
\hline B & 6.85 & 0.96 & 0.23 & 6.78 & 3.50 & 1.67 & 4.66 & 0.88 & 0.19 & 0.105 & 0.05 & \\
\hline A4 & 8.03 & 0.82 & 0.16 & 7.96 & 3.19 & 1.47 & 3.55 & 0.74 & 0.12 & 0.125 & 0.015 & \\
\hline A6 & 8.12 & 0.86 & 0.19 & 8.05 & 3.49 & 1.53 & 3.65 & 0.78 & 0.15 & 0.135 & 0.035 & \\
\hline $\mathrm{S}$ & 9.19 & 0.78 & 0.14 & 9.12 & 2.70 & 1.30 & 3.12 & 0.70 & 0.10 & 0.08 & --- & \\
\hline A9 & 9.03 & 0.80 & 0.16 & 8.96 & 2.67 & 1.31 & 3.37 & 0.72 & 0.12 & 0.095 & --- & \\
\hline A7 & 10.17 & 0.64 & 0.14 & 10.10 & 2.35 & 1.18 & 2.76 & 0.56 & 0.10 & 0.08 & -- & \\
\hline A5 & 10.53 & 0.62 & 0.14 & 10.46 & 2.46 & 1.1 .7 & 2.62 & 0.54 & 0.10 & 0.075 & --- & \\
\hline $\mathrm{X}$ & 11.82 & 0.50 & 0.07 & 11.75 & 1.15 & 0.78 & 1.96 & 0.42 & 0.03 & --- & --- & 1,5 \\
\hline A3 & 11.87 & 0.49 & 0.11 & 11.80 & 1.07 & 0.75 & 1.97 & 0.41 & 0.07 & 0.045 & --- & 5 \\
\hline C & 11.58 & 0.59 & 0.13 & 11.51 & 1.41 & 0.88 & 2.26 & 0.51 & 0.09 & 0.07 & --- & \\
\hline A2 & 11.59 & 0.62 & 0.12 & 11.52 & 1.66 & 0.96 & 2.41 & 0.54 & 0.08 & --- & --- & \\
\hline 18 & 11.55 & 0.57 & 0.09 & 11.48 & --- & 0.75 & 2.25 & 0.49 & 0.05 & --- & --- & 6 \\
\hline 19 & 11.94 & 0.48 & 0.07 & 11.87 & --- & 0.74 & 1.84 & 0.40 & 0.03 & --- & --- & 6 \\
\hline 21 & 9.54 & 0.71 & 0.14 & 9.47 & --- & 1.45 & 2.84 & 0.63 & 0.10 & 0.105 & --- & 3 \\
\hline 30 & 7.80 & 0.88 & 0.18 & 7.73 & --- & 1.49 & 3.59 & 0.80 & 0.14 & 0.155 & -- & \\
\hline 45 & 8.12 & 0.88 & 0.17 & 8.05 & --- & 1.59 & 3.60 & 0.80 & 0.13 & 0.11 & 0.045 & \\
\hline 46 & 8.00 & 0.88 & 0.17 & 7.93 & --- & 1.57 & 3.64 & 0.80 & 0.13 & 0.15 & 0.045 & \\
\hline 75 & 11.96 & 0.66 & 0.07 & 11.89 & --- & 0.95 & 2.24 & 0.58 & 0.03 & --- & --- & 3 \\
\hline 76 & 11.01 & 0.63 & 0.13 & 10.94 & --- & 1.12 & 2.49 & 0.55 & 0.09 & --- & --- & 3 \\
\hline 77 & 8.44 & 0.87 & 0.17 & 8.37 & --- & 1.58 & 3.57 & 0.79 & 0.13 & 0.14 & --- & 3 \\
\hline 78 & 9.30 & 0.72 & 0.15 & 9.23 & --- & 1.34 & 2.87 & 0.64 & 0.11 & 0.08 & --- & 3 \\
\hline 79 & 10.82 & 0.64 & 0.10 & 10.75 & --- & 1.21 & 2.56 & 0.56 & 0.06 & 0.12 & --- & 3 \\
\hline 113 & 8.03 & 0.90 & 0.18 & 7.96 & --- & 1.62 & 3.81 & 0.82 & 0.14 & 0.165 & 0.04 & 2 \\
\hline
\end{tabular}

*Errors are $\pm 0.04, \pm 0.03$, and \pm 0.03 for $J-H, H-K$, and $K$, respectively, unless otherwise noted. Errors are \pm 0.03 or less for $\mathrm{CO}$ and $\mathrm{H}_{2} \mathrm{O}$.

$+U B V$ photometry from $A H$. We assumed a reddening of $E_{B-V}=0.25$. The reddening-corrected $\mathrm{CO}$ and $\mathrm{H}_{3} \mathrm{O}$ indices are 0.005 larger and 0.015 smaller, respectively, than the observed indices.

NOTES: 1. Error is $\pm 0.05 \mathrm{mag}$ in $J-H$.

2. Errors are $\pm 0.05 \mathrm{mag}$ in $J-H$ and $\pm 0.04 \mathrm{mag}$ in $H-K$ and $K$.

3. Errors are $\pm 0.06 \mathrm{mag}$ in $J-H$ and $\pm 0.05 \mathrm{mag}$ in $H-K$ and $K$.

4. Asymptotic giant branch $(\mathrm{AH})$.

5. Horizontal branch (AH) .

6. Probable HB (see Fig. 3 of $\mathrm{AH}$ ).

7. This is a small-amplitude, long-period variable, according to Hogg (1978).

included in the present program. Additional GB and HB stars with photographic $B V$ data from AH's Table 2a were also included.

$$
\text { III. REDDENING CORRECTIONS AND }(m-M)_{0}
$$

Estimates of $E_{B-V}$ for M71 vary from 0.21 to 0.32 mag. Dickens and Rolland (1972) and Kron and Guetter (1976) have quoted the lower value. The former authors based their result on a refitting of $\mathrm{AH}$ 's data to the color-magnitude diagrams of the metal-rich clusters 47 Tuc and NGC 6171. Kron and Guetter compared six-color photometry of M71 with that of other clusters. Racine (1973), on the other hand obtained $E_{B-V}=0.32$ on the basis of mean colors for clusters as functions of Kinman's (1959) integrated spectral types, although such means were poorly determined for the metal-rich clusters. Cuffey (1973) presented $U B V$ magnitudes and colors for 13 stars within several arcmin of M71. By fitting the three apparently early-type main-sequence stars (stars 6,10 , and 12) to a standard $(U-B, B-V)$-diagram, we obtained a mean $E_{B-V}=0.3$. Without a luminosity determination, however, the distances of these stars cannot be derived. We have elected to use an average value of $E_{B-V}=0.25$ but regard it to be uncertain by $\pm 0.05 \mathrm{mag}$. Color excess ratios are those found from van de Hulst's curve 15 (Johnson 1966a); corrections of +0.005 and -0.015 mag have been applied to the $\mathrm{CO}$ and $\mathrm{H}_{2} \mathrm{CO}$ indices, respectively. The reddeningand extinction-corrected colors and magnitudes, denoted by a zero subscript, are given in Table 1 .

Since our adopted value of $E_{B-V}$ differs from that of $\mathrm{AH}$, it is necessary to recalculate $(m-M)_{0}$. If the same technique used by AH is followed [viz., fit the observed main sequence of M71 to a sequence of field stars from Eggen and Sandage 1962 with the same $\delta(U-B)$ and with known distance], the resulting value for $(m-M)_{0}$ is $13.0 \pm 0.2$. Alternatively, we can compare the extinction-corrected horizontalbranch magnitudes of M71, 47 Tuc, and NGC 6171 (Hartwick and Hesser 1974; Sandage and Katem 1964; Lee 1977). In metal-rich cluster, $M_{v}$ for horizontalbranch stars usually lies between 0.6 and 1.0 (the 
TABLE 2

ObServed aNd Derived Parameters for M71 Stars

\begin{tabular}{|c|c|c|c|c|c|}
\hline star & $-B C$ & ${ }^{M} \mathrm{bol}$ & $T_{\text {eff }}$ & $\log \theta$ & Notes \\
\hline $\begin{array}{l}\text { B } \\
\text { A4 } \\
\text { A6 } \\
\text { S } \\
\text { A9 }\end{array}$ & $\begin{array}{l}1.66 \\
0.95 \\
0.97 \\
0.67 \\
0.80\end{array}$ & $\begin{array}{l}-3.13 \\
-2.35 \\
-2.18 \\
-1.34 \\
-1.38\end{array}$ & $\begin{array}{l}3300 \\
4000 \\
3950 \\
4200 \\
4100\end{array}$ & $\begin{array}{l}0.33 \\
0.98 \\
1.02 \\
1.47 \\
1.41\end{array}$ & 2 \\
\hline $\begin{array}{l}\text { N } \\
\text { A7 } \\
\text { A5 } \\
\text { X } \\
\text { A3 }\end{array}$ & $\begin{array}{l}0.21 \\
0.47 \\
0.36 \\
0.13 \\
0.11\end{array}$ & $\begin{array}{l}-0.40 \\
-0.52 \\
-0.17 \\
+0.69 \\
+0.77\end{array}$ & $\begin{array}{l}4900 \\
4500 \\
4600 \\
5200 \\
5200\end{array}$ & $\begin{array}{l}2.12 \\
1.92 \\
2.10 \\
2.65 \\
2.68\end{array}$ & 1 \\
\hline $\begin{array}{l}\text { C } \\
\text { A2 } \\
18 \\
19 \\
21\end{array}$ & $\begin{array}{l}0.25 \\
0.29 \\
0.23 \\
0.06 \\
0.48\end{array}$ & $\begin{array}{l}+0.63 \\
+0.75 \\
+0.61 \\
+0.76 \\
-1.08\end{array}$ & $\begin{array}{l}4850 \\
4700 \\
4900 \\
5300 \\
4400\end{array}$ & $\begin{array}{l}2.51 \\
2.50 \\
2.52 \\
2.71 \\
1.65\end{array}$ & 1 \\
\hline $\begin{array}{l}29 \\
30 \\
45 \\
46 \\
75\end{array}$ & $\begin{array}{l}2.58 \\
0.94 \\
0.92 \\
0.97 \\
0.21\end{array}$ & $\begin{array}{l}-3.39 \\
-2.53 \\
-2.18 \\
-2.31 \\
+1.03\end{array}$ & $\begin{array}{l}3050 \\
3950 \\
3950 \\
3950 \\
4900\end{array}$ & $\begin{array}{l}0.09 \\
0.88 \\
1.02 \\
0.97 \\
2.69\end{array}$ & 1,2 \\
\hline $\begin{array}{r}76 \\
77 \\
78 \\
79 \\
113\end{array}$ & $\begin{array}{r}0.32 \\
0.92 \\
0.51 \\
0.35 \\
1.11\end{array}$ & $\begin{array}{l}+0.22 \\
-1.89 \\
-1.32 \\
+0.07 \\
-2.25\end{array}$ & $\begin{array}{l}4650 \\
4000 \\
4400 \\
4650 \\
3900\end{array}$ & $\begin{array}{l}2.26 \\
1.16 \\
1.56 \\
2.20 \\
0.98\end{array}$ & \\
\hline
\end{tabular}

\footnotetext{
$\mathrm{BC}$ and $M_{\text {bol }}$ are obtained from the optical and infrared photometry with $(m-M)_{0}=12.9 \pm 0.2$. Teff is derived as discussed in the text. Log $g$ is derived from the values of $M_{\mathrm{bol}}$ and $T_{\text {eff, }}$ and by assuming that $M=1 M_{\odot}$ for all the stars.

NOTES: 1 . Only $V-K$ used for $T_{\text {eff }}$ determination.

2. Teff obtained by comparing $(V-K)_{0}$ with Johnson (1966b).
}

above-cited authors; Harris 1976). By using $0.8 \mathrm{mag}$ as a mean, we obtain $(m-M)_{0}=12.9$ for M71 from both 47 Tuc and NGC 6171. This value is adopted here.

\section{BOLOMETRIC CORRECTIONS, EFFECTIVE} TEMPERATURES, AND SURFACE GRAVITIES

Bolometric corrections were computed as in CFP. For stars that did not have observations at $U$, the $U-V$ colors were predicted from a mean $(U-V$, $V-K$ )-relationship. In any case, only a small fraction of the energy from these stars comes out below $4000 \AA$. Since no $R-I$ colors were available for the M71 stars observed, we predicted these colors from the relationships between $V-K, V-R$, and $V-I$ of Johnson (1966b). A comparison of the observed and predicted colors for the stars of CFP which have $R-I$ photometry was made in order to check the accuracy of this procedure. Table 2 gives the final values for the empirically determined bolometric corrections. The absolute bolometric magnitudes $M_{\text {bol }}$ in Table 2 are derived from these corrections-an $E_{B-V}=0.25$, an $(m-M)_{0}=12.9$-and the $V$ magnitudes of $\mathrm{AH}$.

The effective temperatures $T_{\text {eff }}$ were calculated from the model-derived mean relationships between $V-K, J-K$, and $T_{\text {eff }}$ given in CFP. The values of $T_{\text {eff }}$ implied by $V-K$ were given double weight because of the greater sensitivity of this index. Nevertheless, the spread in $T_{\text {eff }}$ values for a given set of $V-K$ and $J-K$ values was generally $100 \mathrm{~K}$ or less. The adopted values for $T_{\text {eff }}$ are given in Table 2. An uncertainty of \pm 0.05 in $E_{B-V}$ affects $\log T_{\text {eff }}$ and $M_{\text {bol }}$ by \pm 0.01 and \pm 0.07 , respectively. Surface gravities for the stars were computed on the assumption that they are all of $1 \mathfrak{M}_{\odot}$. These values are also given in Table 2.

\section{RESULTS}

Most of the reddening- and extinction-corrected data are presented in several figures. We discuss these 


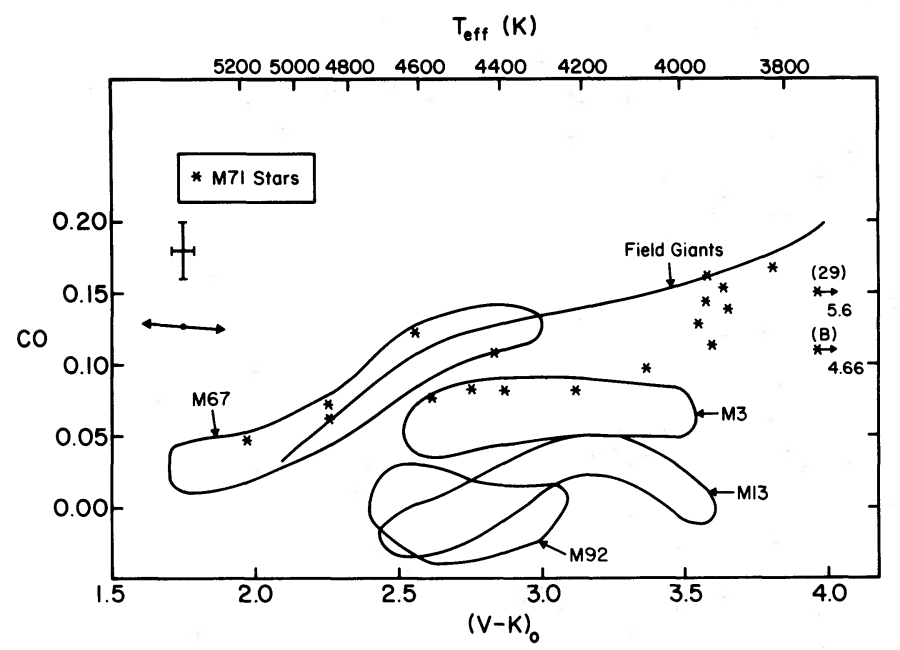

FIG. 1.-The reddening-corrected CO indices and $V-K$ colors are plotted for the M71 stars. Values of $T_{\text {eff }}$ as a function of $V-K$ are from CFP. The error bars show nominal $1 \sigma_{m}$ errors in each coordinate. The effect of a \pm 0.05 uncertainty in $E_{B-V}$ is indicated by the arrows. Regions occupied by the cluster stars observed in CFP are delineated. The numbers near the M71 stars 29 and $B$ are their $(V-K)_{0}$ colors.

first and then turn our attention to a comparison of the $\left(\log L, \log T_{\text {eff }}\right)$-plot with model predictions.

\section{a) $\mathrm{CO}$ and $\mathrm{H}_{2} \mathrm{O}$ Absorption Indices}

Figure 1 shows a plot of $\mathrm{CO}$ strengths versus $(V-K)_{0}$ and $T_{\text {eff }}$. The regions occupied by the stars studied in CFP are indicated. Since M71 is considered to be more metal rich than the globular clusters studied in CFP, it is not surprising that, in the mean, the CO strengths of the giant stars are greater than in the other globulars. Near $(V-K)_{0}=3.00$, the mean CO index for M71 is $0.09 \mathrm{mag}$, with some uncertainty due to either cosmic or observational scatter. Two stars in M71 (B and 29) are 1 and 2 mag redder, respectively, than any of the stars in the other clusters. (Recall that, for the other clusters, the reddest known stars were observed by CFP.) This demonstrates that infrared colors are much more effective than $U B V$ colors in providing temperature resolution at the red end of the giant branch. These two stars have $\mathrm{CO}$ indices 0.07 and $0.10 \mathrm{mag}$, respectively, below the mean field-giant line at the same $(V-K)_{0}$. Star 29 has an $\mathrm{H}_{2} \mathrm{O}$ index of $0.17 \mathrm{mag}$. This value is $0.06 \mathrm{mag}$ greater than the mean for field giants at this $(V-K)_{0}$ and is greater than the $\mathrm{H}_{2} \mathrm{O}$ index for any of the other cluster stars observed by CFP. Such a combination of strong $\mathrm{H}_{2} \mathrm{O}$, weak $\mathrm{CO}$, and red $V-K$ is characteristic of Mira variables (Frogel 1971). For the other stars in Table 1 for which $\mathrm{H}_{2} \mathrm{O}$ was measured, the values of this index are consistent with their $(V-K)_{0}$ colors. On the basis of $4 \mathrm{~m}$ echelle spectra, the radial velocity of M71 is $-25 \pm 2 \mathrm{~km} \mathrm{~s}^{-1}$, in good agreement with that found by Jenner and Kwitter (1977). Of the M stars found near M71 by Stephenson (1961), stars B, 27 (the Mira variable Z Sge), and 46 are definite $V_{r}$ members, while star 29 is a probable member.
Five of the stars in Table 1 (plus 10 from CFP) have also been observed by Pilachowski (1978). Although there is qualitative agreement, quantitative comparison of her results with ours is not possible, because she transformed her observations to a system different from that defined in Paper I. On the basis of her observations of six M71 stars, Pilachowski concluded that the CO indices for M71 and the mean field giants are indistinguishable. However, as discussed above, Figure 1 shows that, for $V-K>2.5$, nearly all of the stars have $\mathrm{CO}$ indices lower than that of the mean field-giant line.

\section{b) Color-Color Plots}

In Figure 2 the $\left[(U-V)_{0},(V-K)_{0}\right]$-plane is reproduced from CFP but with the addition of the M71 stars. The M71 giants tend to lie between the mean field-giant line (or the locus of the M67 stars) and the locus of the metal-poor cluster stars, although the scatter for the M71 stars is greater than that for the other clusters. ${ }^{1}$

For a given $T_{\text {eff }}$, the variation in $(U-V)_{0}$ due to line blanketing as $[\mathrm{Fe} / \mathrm{H}]$ increases is large for $[\mathrm{Fe} / \mathrm{H}]$ between 0.0 and -1.5 but small for $[\mathrm{Fe} / \mathrm{H}]<-1.5$ (CFP). Thus the locations of the M71 stars are consistent with a metallicity for M71 intermediate between that for field giants and M67 on the one hand and that for metal-poor globulars on the other. ${ }^{2}$

\footnotetext{
${ }^{1}$ Such scatter can arise, in part, from photometric problems in the extremely crowded field, nonuniform reddening, or inclusion of nonmembers.

${ }^{2}$ Estimates of the metallicity of M67 have ranged from super-metal rich (Spinrad and Taylor 1969) to somewhat metal poor (Griffin 1975; Bessell 1972; Eggen and Sandage 1964; Eggen 1977). Measurements of CN blanketing on the DDO system of the M67 giants led Janes (1974) to conclude that these stars are between normal field giants and Hyades giants in abundance. A recent photometric investigation of
} 


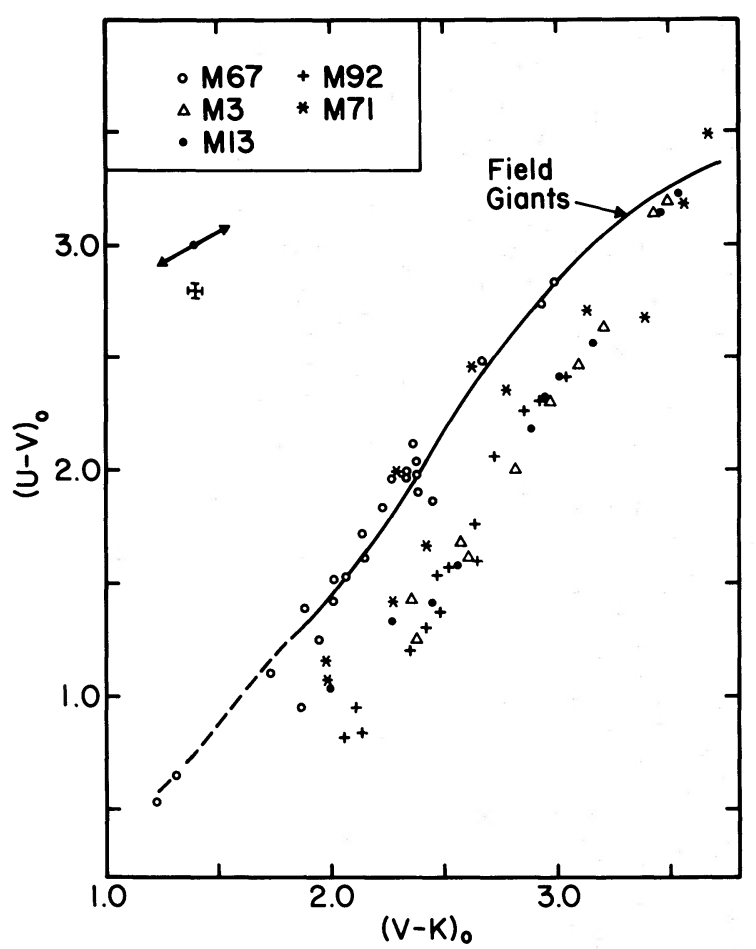

FIG. 2.-The reddening-corrected colors for the M71 stars and all the stars from CFP are shown. The mean field-giant line is from Paper I with the dashed portion from Johnson (1966a). Error bars and reddening vectors are as in Fig. 1.

Adoption of the extremes of the error in $E_{B-V}$ of \pm 0.05 , however, would force the M71 stars to lie close to one sequence or the other.

Figure 3 plots the $\left[(J-H)_{0},(H-K)_{0}\right]$-colors for the M71 stars of Table 1 together with the data from CFP. We see that the M71 stars intermix with the stars from CFP, extend the globular cluster sequence to redder colors, and continue the displacement from the mean relation for field giants. There is an indication that the reddest nonvariable stars in 47 Tuc (Glass and Feast 1973) are also displaced above the mean field-giant line; such a comparison is difficult, however, because of the differences in the photometric systems used. We are unable at present to explain the relative locations of the various clusters in Figure 3.

The locus of points for the M71 stars in the $[(J-$ $\left.K)_{0},(V-K)_{0}\right]$-plane is very similar to that of the metal-poor clusters, which, in turn, is essentially the

the main sequence of several nearby clusters by Taylor (1978) indicates a solar abundance for M67. The CO indices for the M67 stars also imply a metallicity which is indistinguishable from that of the mean field giants, as does the position of the M67 stars in the $(U-V, V-K)$-plot (Fig. 2), which overlaps that of the mean field-giant lines. Thus it is reasonable to conclude that the metallicity of M67 is between 0.0 and -0.15 dex with respect to that of the mean field giants (see $\S$ VI for details).

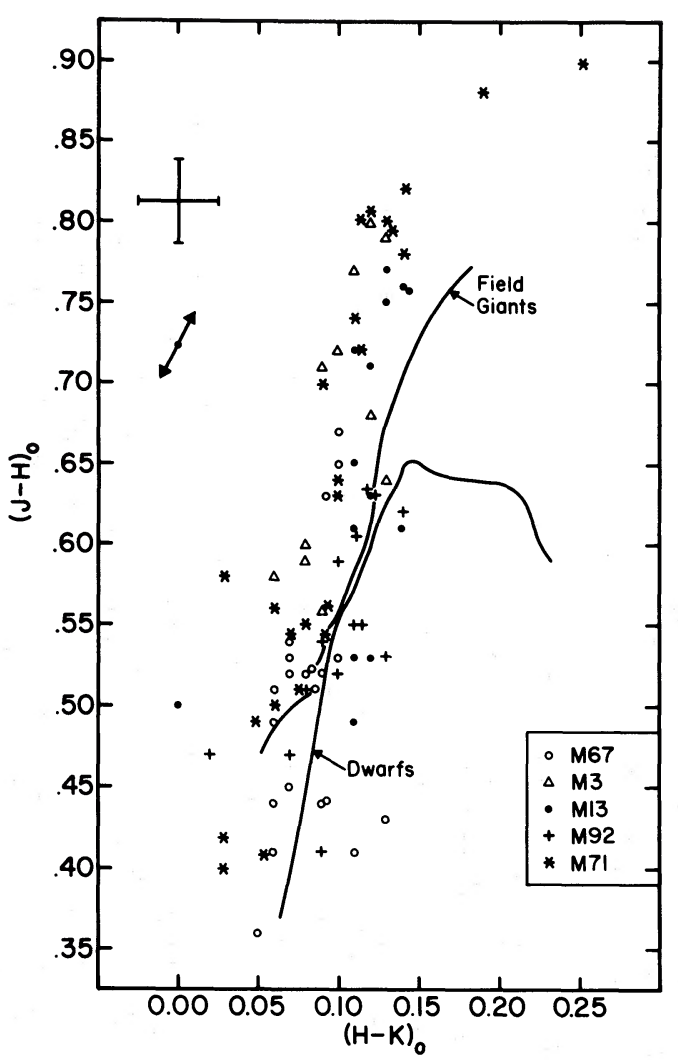

Fig. 3.-Same as Fig. 2

mean locus of field giants. The reader is referred to Figure $2 c$ of CFP for details.

\section{c) $\left(\log L, \log T_{\text {eff }}\right)$-Diagram}

The bolometric luminosities and $T_{\text {eff }}$ (Table 2) are plotted in Figure 4. Also shown are the mean relations for the four clusters from CFP and three evolutionary tracks for metal-deficient stars from Rood (1972). The small, circled area represents the M67 clump stars, which are usually interpreted as the old disk population equivalent of the horizontal branch (e.g., Cannon 1970). The M71 giant branch again lies between the giant branches of the metal-poor clusters and M67, a result that is relatively insensitive to the adopted reddening. This location is consistent with M71 being intermediate in metallicity between M67 and the metalpoor clusters. Globular cluster metallicity estimates based on the relative positions of the red-giant branch are essentially independent of age for ages between 10 and $16 \times 10^{9}$ years (see, e.g., Ciardullo and Demarque 1977).

\section{CNO ABUNDANCES}

We consider in this section what can be deduced about the CNO abundances in M71 and other globular clusters both from the narrow-band $\mathrm{CO}$ photometry presented here and in CFP and from DDO 


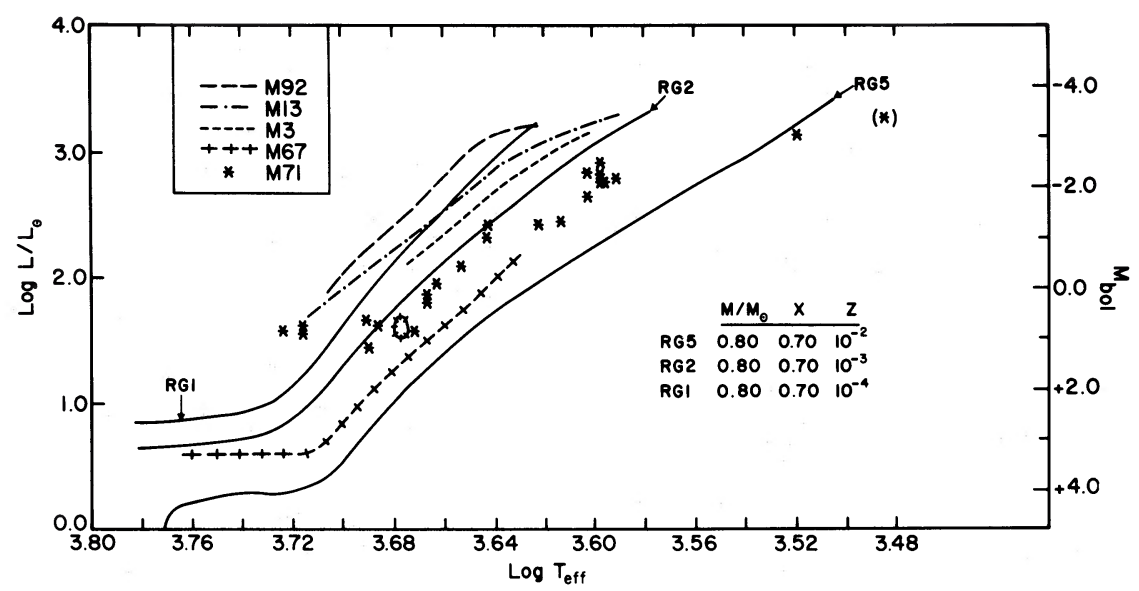

Fig. 4. - The empirical bolometric luminosity-effective temperature plot for the M71 stars. The mean giant branches for the clusters observed by CFP are also plotted. The small, enclosed area represents the M67 clump stars. The evolutionary tracks labeled RG1, RG2, and RG5 are from Rood (1972). Note that the position of the long-period variable star 29 in M71 is uncertain.

photometry. In $\S \mathrm{VI} a$ a procedure for converting observed $\mathrm{CO}$ indices into quantitative measures of the abundances of $\mathrm{C}$ and $\mathrm{O}$ is described. The $\mathrm{C}$ and $\mathrm{O}$ abundances of the M71 stars are derived in $\S \mathrm{VI} b$ and are compared with the qualitative results of $\S \mathrm{V}$. In $\S \mathrm{VI} c$ the $\mathrm{C}$ and $\mathrm{O}$ abundances are derived for $\mathrm{M} 3$, M13, and M92 from the photometric CO indices combined with the results of Cohen (1978). Section VI $d$ discusses the correlation between the CO index and several DDO indices.

\section{a) Procedures}

A method for obtaining quantitative $\mathrm{CO}$ abundances from the $2.4 \mu \mathrm{m} \mathrm{CO}$ index has been developed by using opacity distribution function (ODF) spectra $^{3}$ in this wavelength region from the Bell et al. (1976) and Gustafsson et al. (1975) grids of models for K giants. For a sequence of models of varying $Z$ (defined here so that $\left.Z_{\odot}=1\right)$ at a fixed $T_{\text {eff }}$ and $g$, the mean absorption $A_{\text {co }}$ with respect to an interpolated continuum over the ODF region which includes the first fundamental $\mathrm{CO}$ band was expressed as

$$
A_{\mathrm{Co}}(Z) / A_{\mathrm{Co}^{\odot}}=Z^{\mathrm{MMF}},
$$

where MMF is the metallicity multiplication factor (Cohen 1978). Equation (1) defines a relationship between $\log Z$ and $\log \left[A_{\mathrm{Co}}(Z) / A_{\mathrm{Co}}{ }^{\circ}\right]$ which is similar to that of a classical curve of growth; the function MMF is the slope of the resulting curve at each value of $\log \left[A_{\mathrm{Co}}(Z) / A_{\mathrm{CO}}{ }^{\circ}\right]$. With the use of the models in the Bell and Gustafsson grid (Bell et al. 1976), a curve was plotted of the deduced MMF values as a function of $A_{\mathrm{Co}}$. For different values of $T_{\text {eff }}$ and $\log g$, all the model sequences lie along the same (MMF, $\left.A_{\mathrm{Co}}\right)$-curve shown in Figure 5. For $A_{\mathrm{Co}}$ larger than the maximum of $15 \%$ obtained from the Bell and Gustafsson grid, $\mathrm{MMF}=0.1$ was adopted. For stars of the same $T_{\text {eff }}$

${ }^{3}$ The ODF fluxes for these models were kindly provided by Dr. Bengt Gustafsson. and similar gravities but different $\mathrm{CO}$ absorptions, this curve can be used to derive the difference in CO abundance.

In order to actually use Figure 5 on the observed $2.4 \mu \mathrm{m} \mathrm{CO}$ indices of this paper and of CFP, two corrections must be made. First, the observed CO index is defined with respect to only one continuum point, so it must be corrected for the change in the continuum slope of the observed star as compared with that of Vega, defined to have a CO index of $0.00 \mathrm{mag}$. The maximum correction for giants hotter than $3700 \mathrm{~K}$ is $0.03 \mathrm{mag}$. The observed (reddeningcorrected) $\mathrm{CO}$ index plus this small correction, converted from a magnitude to an absorption, shall be called $A_{\mathrm{co}}{ }^{*}$. Second, the ratio between the "observed"

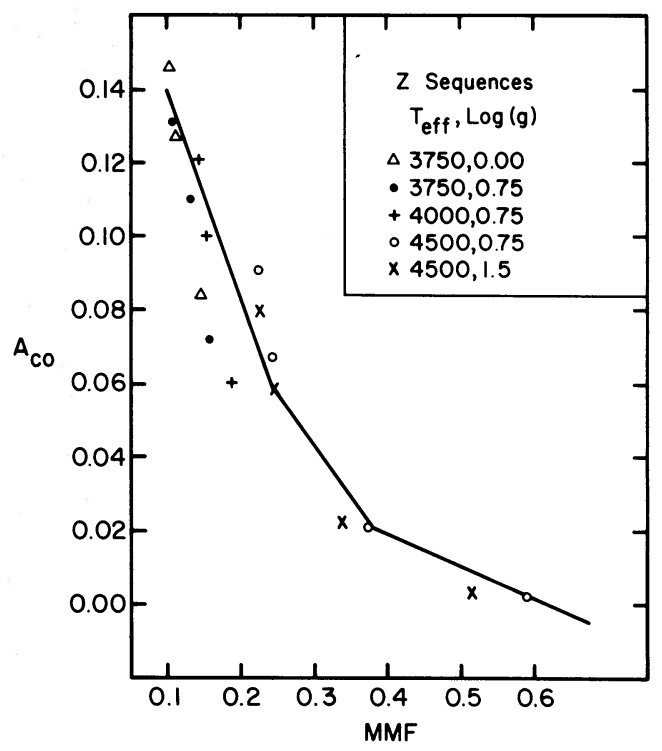

Fig. 5.-Calculated values of $\mathrm{CO}$ absorption as a function of the MMF. See text for description. 
$A_{\mathrm{co}} *$ values for field giants and the theoretically derived $A_{\text {co }}$ values (compared at the same $T_{\text {eff }}$ ) is approximately 1.2 over the range $3750-5000 \mathrm{~K} .^{4}$ Therefore, $A_{\mathrm{co}} *$ is scaled down by this factor.

The Bell and Gustafsson ODF spectra were calculated assuming solar $\mathrm{C} / \mathrm{N} / \mathrm{O} / \mathrm{Fe}$ for $Z=1.0,0.3$, $0.1,0.01$, and 0.001 . Thus an interpolation formula is required for nonsolar $\mathrm{C} / \mathrm{N} / \mathrm{O} / \mathrm{Fe}$. Molecular equilibrium calculations were made by using the code of Kurucz (1970) for the network of molecules involving $\mathrm{C}, \mathrm{N}$, and $\mathrm{O}$. Although the results cannot be directly transformed into photometric indices, they are expected to give the proper sense of the behavior of these indices for stars hotter than $3500 \mathrm{~K}$. The models indicate that, in the temperature range of the grid of CFP, CO is completely associated only in the outermost layers of the atmosphere. For $Z=1-0.01$ and for $\mathrm{C} / \mathrm{O}<1$, the simplest interpolation formula that fits the molecular equilibrium calculations of the $\mathrm{CO}$ column density through the atmosphere above $\tau(1 \mu \mathrm{m})=0.3$ is $N(\mathrm{CO}) \propto N(\mathrm{C}) \times\left[N(\mathrm{O})^{1 / 2}\right]$. This formula is accurate to within 0.1 dex for $C, N$, and $O$ varied by factors smaller than 3 from their relative solar values. For $\mathrm{CN}$, the situation is simpler, and a suitable interpolation formula is $N(\mathrm{CN}) \propto N(\mathrm{C}) \times$ $N(\mathrm{~N})$ for small relative variations in $\mathrm{C}, \mathrm{N}$, and $\mathrm{O}$ abundances.

Thus, in principle, an observed $\mathrm{CO}$ index can yield only the product $N(\mathrm{C}) \times[N(\mathrm{O})]^{1 / 2}$, while the observed DDO CN index could determine the product of the $\mathrm{C}$ and $\mathrm{N}$ abundances. When all of the uncertainties are considered, the resultant products of abundances for individual stars have an accuracy of \pm 0.4 dex. Thus the analysis can be used meaningfully only in a statistical sense to obtain the mean $\mathrm{C} \times \mathrm{O}^{1 / 2} /(\mathrm{C} \times$ $\left.\mathrm{O}^{1 / 2}\right)_{\odot}$ for a group of stars in a cluster.

\section{b) $M 71$}

Before applying the above techniques to M71, we note that, because of the effects of convective mixing in evolved stars, those stars which are used to define the mean field-giant line (cf. Figs. 1 and 2) and which are assumed to have solar $\mathrm{Fe} / \mathrm{H}( \pm 0.15 \mathrm{dex})$ probably do not have solar CNO abundances. An observational study of CNO ratios among bright field giants by Lambert and Ries (1977) shows that they have solar oxygen abundance, $[\mathrm{C}]=-0.25$ dex and $[\mathrm{N}]=$ $+0.35 \mathrm{dex} ;^{5}$ we shall henceforth assume that their results apply to the mean field-giant line shown in Figures 1 and 2. These changes from solar ratios are in qualitative agreement with theoretical predictions by Dearborn, Bolton, and Eggleton (1975) of the effects of convective mixing in evolved stars of solar metallicity. Such predictions for the effects of mixing

${ }^{4}$ Such a discrepancy could arise from incorrect transition probabilities for the $\mathrm{CO}$ band chosen by Bell and Gustafsson. Nonsolar CNO ratios due to convective mixing are in the wrong sense and cannot eliminate the necessity for this correction (see $\S \mathrm{VI} b$ ).

${ }^{5}[X]=\log (X / H)_{\text {star }}-\log (X / H)_{\odot}$. on CNO ratios in metal-poor stars, although urgently needed, are not available. However, from the observations of Sneden (1974), a similar enhancement of $N$ and decrease in $\mathrm{C}$ is found in metal-poor $\mathrm{K}$ giants as compared with metal-poor $\mathbf{K}$ dwarfs. Furthermore, there is some evidence that metal-poor field $\mathrm{K}$ giants have $[\mathrm{O} / \mathrm{Fe}]$ larger than solar (Lambert, Sneden, and Ries 1974; Conti et al. 1967).

By using the analysis of the $\mathrm{CO}$ index discussed in $\S \mathrm{VI} a$, we derive $\left[\mathrm{C} \times \mathrm{O}^{1 / 2}\right]_{m f}$, where $\mathrm{mf}$ refers to the mean of bright field giants, not the Sun, and is used for $\mathrm{CNO}$ only. We define $\left[\mathrm{C} \times \mathrm{O}^{1 / 2}\right] / 1.5$ as $[\mathrm{C} * \mathrm{O}]$, where the denominator is a normalization factor used to make [C * O] numerically directly comparable to the conventional $[\mathrm{Fe}$ ], so that we derive from 17 stars in M71 (excluding the two extremely red stars) $[\mathrm{C} * \mathrm{O}]=-0.4 \pm 0.2$ dex. Table 3 summarizes estimates for the metallicity of M71. The first four values are based on the measurement of the effect of heavy metals on the spectra of individual stars, while the last two involve the $\mathrm{CNO}$ abundances. ${ }^{6}$ In summary, it is reasonable to conclude that the heavy-metal abundance of M71 is a factor of 2 down from solar with an uncertainty of perhaps $\pm 0.2 \mathrm{dex}$. The $\mathrm{CO}$ results are in good agreement with the other metallicity determinations, thus implying that the M71 stars may have the same $\mathrm{C} / \mathrm{O} / \mathrm{Fe}$ as the mean field $\mathrm{K}$ giants used to calibrate the narrow-band system. ${ }^{7}$

The above results are in agreement with the qualitative conclusions of $\S \mathrm{V}$ where we compared M67 and M71, with the assumption that M67 has near-solar abundances. If the lower metallicities suggested for M67 by Griffin (1975), Bessel (1972), and Eggen (1977) are correct, however, M67 will have an anomalously high ( $\mathrm{CO}$ and $\mathrm{CN}) / \mathrm{Fe}$ ratio, about 0.4 dex higher than that of the mean field giants and than that of M71. Unfortunately, the available analyses are too limited to allow an accurate determination of the heavy-element abundance of M67 relative to M71. A detailed abundance analysis of stars in these two clusters is in progress by one of us (J. G. C.).

\footnotetext{
${ }^{6}$ Although the values are certainly in agreement, in view of the quoted errors, those two which indicate a solar abundance warrant some further comments. Butler's (1975) determination is based on only one star: one of AH's "blue stragglers," which is assumed to be a cluster member and is also assumed to have an atmospheric structure similar to that of the RR Lyrae stars upon which the $\Delta S$ calibration is based. HHM's determination of $[\mathrm{Fe} / \mathrm{H}]$ is based on a similarity of heavy-element blanketing, as determined from DDO photometry, in M71 and NGC 6352 and on the abundance of NGC 6352 derived by Hartwick and Hesser $(1972 b)$ of $[\mathrm{Fe} / \mathrm{H}]=+0.1+0.1$ based on $\delta(U-B)$. However, inspection of HHM's Figure $1 d$ would not seem to rule out a $C_{0}(38-42)$ blanketing for M71 several hundredths of a magnitude less than that for NGC 6352. The measurement of the CN strengths via DDO photometry could not be used for a metallicity determination in these clusters because of the large scatter in this index (HHM).

${ }^{7}$ Since only the product $\left[\mathrm{C} \times \mathrm{O}^{1 / 2}\right]$ is determined, there are $\mathrm{C} / \mathrm{O}$ ratios different from that of the mean field giants that will produce the observed $\mathrm{CO}$ index; these cannot be eliminated without additional data.
} 
TABLE 3

Metallicity Determinations FOR M71

\begin{tabular}{|c|c|c|}
\hline$[\mathrm{Fe} / \mathrm{H}]$ & Method & Source \\
\hline$-0.3 \pm 0.3$ & $\delta(U-B)$ & Arp and Hartwick (1971) \\
\hline$-0.04 \pm 0.2$ & $\Delta S$ & Butler (1975) \\
\hline$-0.3 \pm 0.3$ & $\left(\mathrm{C}, \mathrm{M}, \mathrm{T}_{1}, \mathrm{~T}_{2}\right)$ & Canterna (1975) \\
\hline 0.0 & DDO & HHM \\
\hline$-0.4 \pm 0.2 *$ & TiO & Mould and McElroy (1978) \\
\hline$-0.4 \pm 0.2 t$ & $\mathrm{CO}$ & This paper \\
\hline$-0.2 \pm 0.1$ & $\begin{array}{l}\text { Line blanketing in the } \\
\text { blue calibrated to } \Delta S\end{array}$ & Searle and Zinn (1978) \\
\hline
\end{tabular}

*Also defined with respect to the mean of bright field giants. tThis is $[C * 0]_{\mathrm{mf}}$, defined with respect to the mean of bright field giants; $[\mathrm{C} * 0]^{\prime} \approx-0.6 \pm 0.2$ with respect to the sun (see Lambert and Ries 1977).

c) $M 3, M 13$, and $M 92$

The narrow-band CO measurements of CFP for stars in M3, M13, and M92 can be converted into $[\mathrm{C} * \mathrm{O}]$ abundances via the techniques described above. Table 4 displays the deduced mean $[\mathrm{C} * \mathrm{O}]_{m f}$ for each globular cluster. Column (2) gives the mean $[\mathrm{C} * \mathrm{O}]_{m f}$ deficiency with respect to the mean field giants. Column (3) corrects the mean field giants to the Sun by using the results of Lambert and Ries (1977). Column (4) lists the spectroscopically determined [O] with a correction for $\mathrm{O}$ in $\mathrm{CO}$ based on the $[\mathrm{C} * \mathrm{O}]$ deduced from the observed $\mathrm{CO}$ (this paper; Cohen 1978). Column (5) contains [C] obtained from combining the $\mathrm{CO}$ and $\mathrm{O}$ determinations, i.e., $[\mathrm{C}]=$ $1.5 \times[\mathrm{C} * \mathrm{O}]-0.5[\mathrm{O}]$ (deduced from cols. [2] and [4]). Column (6) is the spectroscopic Fe abundance (Cohen 1978). Although [Fe] is larger in M13 than in $\mathrm{M} 3,[\mathrm{C}]$ and $[\mathrm{C} * \mathrm{O}]$ are larger in M3 than in M13. This result is consistent with the discussion in CFP, Cohen (1978), and Pilachowski (1978). The rather large errors preclude any further discussion of $\mathrm{C}$ and $\mathrm{O}$ abundances.

\section{d) Comparison with DDO Photometry}

Figures $6 a$ and $6 b$ compare the $\mathrm{CO}, \mathrm{CN}\left[C_{0}(41-\right.$ $42)]$, and DDO metal-blanketing index $\left[C_{0}(38-42)\right]$ for individual stars from Table 1 here; from CFP; and from Osborn (1973), Janes (1974), and HHM. Additional new DDO photometry is given in the Appendix. Figure $6 a$ shows that there is a general trend for the abundances of $\mathrm{Fe}, \mathrm{Ti}$, and the other elements which strongly contribute to ultraviolet line blanketing to be correlated with the $\mathrm{CO}$ abundance. In Figure $6 a$ the M13 stars, as expected, show weak CO for their ultraviolet blanketing. Two moderately discrepant M92 points are III-13 and VII-18, both of which have an ultraviolet color too red for the $\mathrm{CO}$ strengths. These are the coolest giants in M92 observed on both systems, and the excessively red ultraviolet color index could be due to Rayleigh scattering rather than line blanketing. The same effect can be seen in Figure 2: for the coolest giants the ultraviolet excess of the M92 giants is less than would be expected when compared with the M3 or M13 giants of the same ( $V-$ $K)_{0}$, while for the hotter giants the M92 stars have

TABLE 4

C ANd O Abundances for Giants in Metal-poor Globulars

\begin{tabular}{|c|c|c|c|c|c|}
\hline & $\begin{array}{c}{[\mathrm{c} * \mathrm{O}]_{\mathrm{mf}}} \\
(\mathrm{dex})\end{array}$ & $\begin{array}{c}{[c * 0]} \\
(\mathrm{dex})\end{array}$ & $\underset{(\mathrm{dex})}{[\mathrm{corr}}$ & $\begin{array}{c}{[c]} \\
(\operatorname{dex})\end{array}$ & $\begin{array}{l}{[\mathrm{Fe}]} \\
(\mathrm{dex})\end{array}$ \\
\hline M3 & $-1.2 \pm 0.3$ & $-1.35 \pm 0.3$ & $-1.4 \pm 0.3$ & $-1.3 \pm 0.4$ & $-1.8 \pm 0.1$ \\
\hline M13 & $-1.95 \pm 0.3$ & $-2.1 \pm 0.3$ & $\leq-1.4 \pm 0.3$ & $z-2.4 \pm 0.4$ & $-1.6 \pm 0.1$ \\
\hline M92 & $s-2.1 \pm 0.3$ & $s-2.3 \pm 0.3$ & & & $-2.3 \pm 0.1$ \\
\hline Source & $\mathrm{CO}(\mathrm{CFP})$ & $\begin{array}{c}\text { Co + Lambert } \\
\text { and Ries (1977) }\end{array}$ & $\begin{array}{l}\text { Cohen }(1978) \\
+[C * 0]\end{array}$ & $\begin{aligned} & {[\mathrm{O}] \operatorname{corr} } \\
&+[\mathrm{C} * \mathrm{O}]\end{aligned}$ & Cohen (1978) \\
\hline
\end{tabular}




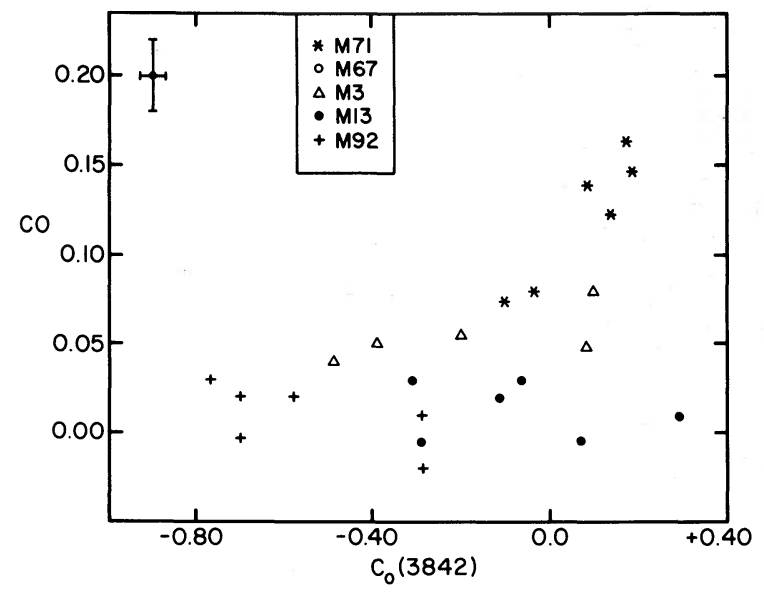

Fig. $6 a$

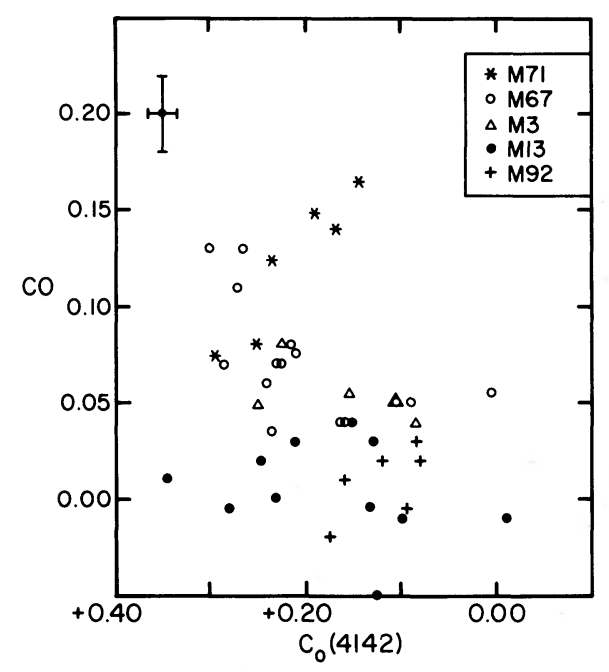

FIG. $6 b$

FIG. 6.-The reddening-corrected $\mathrm{CO}$ indices from this paper and from CFP are plotted against the DDO indices which measure the effects $(a)$ of metal blanketing and $(b)$ of $\mathrm{CN}$ absorption. Error bars are $1 \sigma_{m}$.

larger ultraviolet excesses than those of similar M3 and M13 giants.

In Figure $6 b$ consider first the locations of the stars in the metal-poor clusters-M92, M13, and M3. As pointed out by HHM, these stars have no observable $\mathrm{CN}$. In fact, it can be shown that, for these stars, the dependence of $C_{0}(41-42)$ as a function of temperature as measured by $C_{0}(45-48)(\mathrm{HHM})$ or $(V-K)_{0}$ (CFP and here) is due largely to the change in continuum slope as $T_{\text {eff }}$ changes. Thus, for M3, M13, and M92, Figure $6 b$ is really displaying only the variation of the observed $\mathrm{CO}$ strengths. We note, however, that M13 IV-25 (Appendix) has a $C_{0}(41$ 42) which is about 0.1 mag greater than expected for its $T_{\text {eff }}$ as measured by its $(V-K)_{0}$, for example. Perhaps this is a mild example of the phenomena observed in several other clusters by HHM and by Hesser, Hartwick, and McClure (1976).
Now consider M67 and M71, the two most metalrich clusters in this figure. Taken together, the stars from these clusters define a sequence of monotonically increasing $\mathrm{CO}$ but which exhibits a definite peak in the CN strength. Recall that, in normal field giants, $\mathrm{CN}$ absorption reaches a maximum at about $\mathrm{K} 3$ and then declines for later spectral type (e.g., Griffin and Redman 1960). K3 corresponds to $V-K=2.9$, $\mathrm{CO}=0.12$, and $T_{\text {eff }} \approx 4400 \mathrm{~K}$ (Paper I; Fig. 1 here). In order to investigate the dependence of $\mathrm{CN}$ absorption on $Z$ and $\mathrm{CO}$, the values of $N(\mathrm{CO})$ and $N(\mathrm{CN})$ [the column densities of $\mathrm{CO}$ and $\mathrm{CN}$ above $\tau(1 \mu \mathrm{m})=0.3$ ] were predicted from the molecular equilibrium calculations of $\S$ VI $a$. Figure 7 shows three sequences of $N(\mathrm{CO})$ versus $N(\mathrm{CN})$ corresponding to three values of $Z / Z_{\odot}$. The dependence of $N(\mathrm{CN})$ on $N(\mathrm{CO})$ in Figure 7 is qualitatively similar to that seen for the M67 and M71 stars in Figure $6 b$. Note that, for $Z / Z_{\odot}=1$, the maximum value of $N(\mathrm{CN})$ occurs at about $4500 \mathrm{~K}$, in agreement with the observed maximum absorption strength at $\mathrm{K} 3$ and with other such calculations (see, e.g., Dolan 1965). Also, the maximum value of $N(\mathrm{CN})$ occurs in stars of cooler $T_{\text {eff }}$ as $Z / Z_{\odot}$ is reduced.

In Figure $6 b$ the M67 stars define a line of CO increasing with $\mathrm{CN}$; the coolest stars (those which have the largest $\mathrm{CO}$ indices) have $T_{\text {eff }}=4300 \mathrm{~K}$ (CFP), not quite cool enough for the falloff in $\mathrm{CN}$ to begin. However, for M71, the coolest stars have $T_{\text {eff }}=3800 \mathrm{~K}$ (Table 2), and the CN decline is apparent. Since the observed values of $C_{0}(41-42)$ for the metal-poor clusters are influenced primarily by the continuum slope rather than by true $\mathrm{CN}$ differences, no meaningful comparison with Figure 7 can be made for these stars. Thus it is possible that a small part of the dispersion in $\mathrm{CN}$ indices in Figure 1 of HHM is due to the CN turnover shown in Figure 7. This is true only for the coolest stars in the more metal-rich clusters such as 47 Tuc and M71.

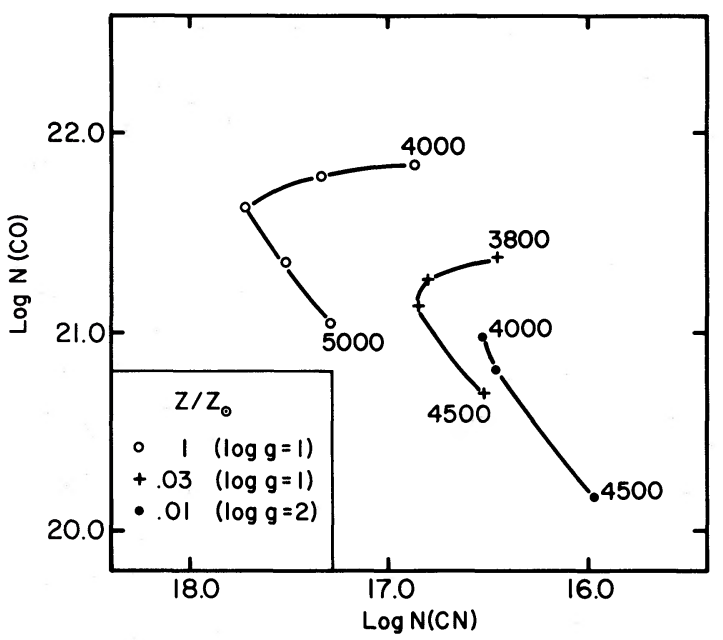

Fig. 7.- Column densities of $\mathrm{CO}$ and $\mathrm{CN}$ predicted by molecular equilibrium calculations for sequences of models of varying $Z / Z_{\odot}$. The highest and lowest temperatures for each sequence are indicated. 


\section{SUMMARY AND CONCLUSIONS}

We have presented observations of $J H K$ colors and $\mathrm{CO}$ and $\mathrm{H}_{2} \mathrm{O}$ line indices for a selection of giant and horizontal-branch stars in the strong-lined globular cluster M71. These data are used to derive $M_{\text {bol }}$ and $T_{\text {eff }}$ for the stars. A theoretical calibration, based on the Bell and Gustafsson (Bell et al. 1976) ODF spectra, is presented for the narrow-band $\mathrm{CO}$ index. The product of $N(\mathrm{C})$ and $[N(\mathrm{O})]^{1 / 2}$ is deduced from the observations of the M71 stars and those stars considered in CFP. A theoretical analysis of the expected behavior of $\mathrm{CO}$ and $\mathrm{CN}$ as a function of $T_{\text {eff }}$ and metallicity is also described. The basic results of this work can be summarized as follows:

1. In a $(U-V, V-K)$-plot the M71 stars lie intermediate between the metal-poor globulars and the locus of solar-neighborhood field giants.

2. In spite of the fact that its population is considerably sparser than that of the metal-poor clusters, M71 has a giant branch which extends to considerably cooler $T_{\text {eff }}$.

3. A $\left(\log L, \log T_{\text {eff }}\right)$-plot is in qualitative agreement with Rood's (1972) red-giant evolutionary tracks. However, detailed comparison with stellar models is difficult because of the problems of convection and the possible sensitivity of these evolutionary tracks to variations in the $Z_{\mathrm{CNO}} / Z$ ratio.

4. The abundance of the M71 giants as deduced from the $\mathrm{CO}$ indices indicates a metal deficiency of -0.4 dex in the mean with respect to field giants. For the M3, M13, and M92 giants, CO data and spectroscopic $\mathrm{O}$ abundances yield $\mathrm{C}$ abundances which support the skewed abundance distribution for M3 and M13 discussed by Cohen (1978).
5. A comparison of our $\mathrm{CO}$ data with published $\mathrm{CN}$ data (on the DDO system) indicates that, if the metallicity of M67 is solar, the photometric indices are consistent with the giants in M92, M3, and M71 having the same $\mathrm{C} / \mathrm{O}$ ratios as the $\mathrm{M} 67$ giants and the mean field giants (to within a factor of 3 ). If the metallicity $(\mathrm{Fe} / \mathrm{H})$ of $\mathrm{M} 67$ is below solar, near that of $\mathrm{M} 71$, then $\mathrm{CNO} / \mathrm{Fe}$ is higher in M67 than in M71.

6. $\mathrm{M} 3$ has an abnormally large $\mathrm{CNO} / \mathrm{Fe}$ ratio as compared with M13; but compared with the mean field giants at solar metallicity, we cannot determine from the photometry alone which is the peculiar cluster.

7. The behavior of the $\mathrm{CN}$ and $\mathrm{CO}$ column densities as a function of $T_{\text {eff }}$ and metallicity can explain the dependence of $C_{0}(41-42)$ on $T_{\text {eff }}$ for the cooler stars in the more metal-rich clusters.

8. For the clusters of CFP and the present paper, the data do not yet show signs of the discontinuity between globular clusters and old disk open clusters which is noted in other studies (e.g., Hartwick and Hesser 1972a; Demarque and McClure 1977), although this conclusion is sensitive to the adopted values of the reddening.

We thank Juan Carrasco and Gary Tuton for their assistance at the telescope. J. A. F. is grateful to Hale Observatories for guest observer privileges during the several observing runs required for this globular cluster project. We have had several informative conversations with Jeremy Mould and Olin Eggen. Several of our colleagues at CTIO have commented on an earlier draft of this paper. This work was supported in part by NSF grant AST 74-18555A2 and NASA grant NGL 05-002-207.

TABLE 5

DDO PHOTOMETRY OF M3, M13, AND M71 StaRS

\begin{tabular}{llll}
\hline & $C$ (3841) & $C$ (4142) & $C$ (4245) \\
\hline$\underline{M}$ & & & \\
AA & -0.167 & 0.252 & 1.047 \\
M13 & & & \\
IV-25 & -0.040 & 0.345 & 1.115 \\
II-90 & -0.352 & 0.249 & 1.009 \\
II-76 & -0.431 & 0.130 & 0.826 \\
III-18 & -0.414 & 0.134 & 0.859 \\
III-63 & -0.266 & 0.211 & 0.960 \\
I-18 & & -0.012 & 0.660 \\
I-23 & & 0.126 & 0.664 \\
I-24 & & 0.099 & 0.785 \\
I-48 & & 0.232 & 0.939 \\
II-67 & & 0.284 & 1.055 \\
III-73 & & 0.165 & 0.840 \\
M71 & & & \\
A4 & & & \\
46 & -0.029 & 0.251 & 1.266 \\
& +0.069 & 0.205 & 1.319 \\
\hline
\end{tabular}




\section{APPENDIX}

Additional DDO photometry for M3, M13, and M71 stars with infrared CO observations from CFP or the present work was obtained with the $1.5 \mathrm{~m}$ telescope at KPNO during 1978 May-June using the Mark II computer photometer and the fainter G-M standard stars from McClure (1976). M13 I-24, II-90, and IV-25 were observed on two nights each; the remaining stars were observed only once. The photometric errors of the photometry listed in Table 5 are $\pm 0.02 \mathrm{mag}$.

\section{REFERENCES}

Aaronson, M., Frogel, J. A., and Persson, S. E. 1978, Ap. J., 220, 442 (Paper II).

Arp, H. C., and Hartwick, F. D. A. 1971, Ap. J., 167, 499 (Ä).

Bell, R. A., Eriksson, K., Gustafsson, B., and Nordlund, A. 1976, Astr. Ap. Suppl., $23,37$.

Bessell, M. S. 1972, Pub. A.S.P., 84, 489.

Butler, D. 1975, Ap. J., 200, 68.

Cannon, R. D. 1970, M.N.R.A.S., 150, 111.

Canterna, R. 1975, Ap. J. (Letters), 200, L63.

Ciardullo, R. B., and Demarque, P. 1977, Trans. Astr. Obs. Yale Univ., Vol. 35.

Cohen, J. G. 1978, Ap. J., 223, 487.

Cohen, J. G., Frogel, J. A., and Persson, S. E. 1978, Ap. J., 222, 165 (CFP).

Conti, P., Greenstein, J. L., Spinrad, H., Wallerstein, G., and Vardya, M. A. 1967, Ap. J., 148, 105.

Cuffey, J. 1973, A.J., 78, 408.

Dearborn, D. S., Bolton, A. J. C., and Eggleton, P. P. 1975, M.N.R.A.S., 170, 7P.

Demarque, P., and McĆlure, R. D. 1977, Ap. J., 213, 716.

Dickens, R. F., and Rolland, A. 1972, M.N.R.A.S., 160, 37.

Dolan, J. F. 1965, Ap. J., 142, 1621.

Eggen, O. J. 1977, Ap. J., 215, 812.

Eggen, O. J., and Sandage, A. R. 1962, Ap. J., 136, 735.

- 1964, Ap. J., 140, 130.

Frogel, J. A. 1971, Ph.D. thesis, California Institute of Technology.

Frogel, J. A., Persson, S. E., Aaronson, M., and Matthews, K. 1978, Ap. J., 220, 75 (Paper I).

Glass, I. S., and Feast, M. W. 1973, M.N.R.A.S., 163, 245.

Griffin, R. 1975, M.N.R.A.S., 171, 181

Griffin, R. F., and Redman, R. O. 1960, M.N.R.A.S., 120, 287.

Gustafsson, B., Bell, R. A., Eriksson, K., and Nordlund, A. 1975, Astr. Ap., 42, 407.
Harris, W. E. 1976, A.J., 81, 1095.

Hartwick, F. D. A., and Hesser, J. E. 1972a, Pub. A.S.P., 84,813 . 1972b, Ap. J., 175, 77. 1974, Ap. J. (Letters), 194, L129.

Hesser, J. E., Hartwick, F. D. A., and McClure, R. D. 1976, Ap. J. (Letters), 207, L113.

Ap. 1977, Ap. J. Suppl., 33, 471 (HHM).

Hogg, H. S. 1978, private communication.

James, K. A. 1974, Ap. J., 189, 423.

Jenner, D. C., and Kwitter, K. B. 1977, Bull. AAS, 9, 287.

Johnson, H. L. 1966a, in Nebulae and Interstellar Matter, ed. B. M. Middlehurst and L. M. Aller (Ch icago: University of Chicago Press), p. 167.

- 1966b, Ann. Rev. Astr. Ap., 4, 193.

Kinman, T. 1959, M.N.R.A.S., 119, 538.

Kron, G. E., and Guetter, H. H. 1976, A.J., 81, 817.

Kurucz, R. L. 1970, Smithsonian Ap. Obs.' Spec. Rept., No. 309.

Lambert, D. L., and Ries, L. M. 1977, Ap. J., 217, 1508.

Lambert, D. L., Sneden, C., and Ries, L. M. 1974, Ap. J., 188, 97.

Lee, S. W. 1977, Astr. Ap. Suppl., 27, 381.

McClure, R. D. 1976, A.J., 81, 182.

Morgan, W. W. 1959, A.J., 64, 432.

Mould, J. R., and McElroy, D. B. 1978, Ap. J., 221, 580.

Osborn, W. 1973, Ap. J., 186, 725.

Pilachowski, C. A. 1978, Ap. J., 224, 412.

Racine, R. 1973, A.J., 78, 180

Rood, R. T. 1972, Ap. J., 177, 681.

Sandage, A., and Katem, B. 1964, Ap. J., 139, 1088.

Searle, L., and Zinn, R. 1978, preprint.

Sneden, C. 1974, Ap. J., 189, 493.

Spinrad, H., and Taylor, B. J. 1969, Ap. J., 157, 1279.

Stephenson, J. B. 1961, A.J., 66, 85.

Taylor, B. J. 1978, Ap. J. Suppl., 36, 173.

Judith G. Cohen: Kitt Peak National Observatory, P.O. Box 26732, Tucson, AZ 85726

JAY A. Frogel: Cerro Tololo Inter-American Observatory, Casilla 63-D, La Serena, Chile

S. E. Persson: Hale Observatories, 813 Santa Barbara Street, Pasadena, CA 91106 